\title{
Filtering Mixing Planes for Low Reduced Frequency Analysis of Turbomachines
}

\author{
G.Pullan and J.J. Adamczyk* \\ Whittle Laboratory \\ University of Cambridge \\ 1 JJ Thomson Avenue \\ Cambridge, CB3 ODY, UK \\ Email: gp10006@cam.ac.uk
}

\begin{abstract}
A class of problems in turbomachinery is characterized by unsteady interactions at low reduced frequencies. These interactions are often the result of perturbations with length-scale on the order of the machine circumference and examples include axial compressors operating with inlet distortion, fans with downstream pylons, and turbine rotors downstream of mid-frame struts. Typically, this unsteadiness is accompanied by higher frequency fluctuations caused by perturbations with a length-scale on the order of a blade pitch. Conventional numerical analysis of this class of problem requires computations with a time step governed by the high frequency content but a greatly reduced run time could be achieved if the time step were dictated solely by the low reduced frequency, long lengthscale, interaction of interest.

In this paper, a filtering mixing plane technique is proposed that removes unwanted short length-scale perturbations at the interfaces between blade rows. This approach gives the user control over the amount of mixing that occurs at these interfaces with the limits being fully mixed-out to pitchwise uniformity (conventional mixing plane) or no mixing (conventional sliding plane). By choosing to retain only enough harmonics to resolve the low reduced frequency interaction of interest, an order of magnitude reduction in run time can be achieved.
\end{abstract}

\section{INTRODUCTION}

Turbomachines are inherently unsteady. Unsteady Reynolds-averaged Navier-Stokes (U-RANS) solvers are widely used to capture the unsteadiness caused by blade passing. This unsteadiness is deterministic with a length-scale on the order of a blade pitch. U-RANS codes will also capture non-deterministic unsteadiness such as that caused by trailing edge vortex

*Visiting Researcher 
shedding, shear layer instability or stall cell propagation, provided that the time step employed is small enough to resolve the frequencies involved. These solvers can also be used to capture unsteadiness with a longer length-scale, on the order of the circumference of the machine, but these simulations are computationally expensive. The origin of the challenge is that the solvers require a time step set by the dominant frequency of unsteadiness (blade passing frequency) but the frequency of interest is much lower. In this paper, we propose a spatial filtering approach to remove the unwanted high frequencies, leaving the user free to select a larger time step to capture the long length-scale unsteady flow features of interest.

The parameter that characterises the class of problems under consideration is the reduced frequency,

$$
\beta=\frac{f}{V_{x} / c_{x}}
$$

where $f$ is the frequency of the unsteadiness, $V_{x}$ is a representative axial velocity and $c_{x}$ the axial chord. If $\beta \ll 1$ the flow is quasi-steady; unsteady effects become important when $\beta \approx 1$ and are likely to dominate at $\beta \gg 1$ (It has also been demonstrated [1] that turbine wakes expected to have a high value of $\beta$ can mix and merge prior to the leading edge of the next blade row, leading to a limited unsteady interaction). For a frequency arising from a circumferential perturbation of length-scale $\lambda$ and blade speed $U$, the reduced frequency is given by:

$$
\beta=\frac{1}{\phi} \frac{c_{x}}{\lambda}
$$

where $\phi$ is the flow coefficient. Taking $\phi \approx 0.5$ and $c_{x} / \lambda \approx 0.01$ for a core compressor with a once-per-rev inlet distortion gives $\beta \approx 0.02$ and we expect quasi-steady behavior in the compressor rotor rows. In certain design situations, such as non-axysmmetric intake design or the arrangement of a non-uniform row of stator blades, the primary concern of the aerodynamicist is the transfer of the long length-scale perturbation and it is this type of low reduced frequency analysis that is the motivation for the current work.

Using a "direct unsteady" U-RANS approach to compute low reduced frequency problems is feasible but wasteful of computational resource as reduced frequencies of order 1 are likely to be resolved. Frequency domain methods, as developed by He and Ning [2] and Hall et al. [3] are an efficient alternative for low reduced frequency problems. A thorough review of these methods is provided by $\mathrm{He}$ [4]. As well as the disconnect between the frequency domain approach and the time-domain methods that are prevalent in turbomachinery aerodynamic analyses, an additional challenge is that the user must prescribe the set of frequencies to be resolved; this is particularly challenging for a domain comprized of many stages or that contains unsteadiness at non-deterministic frequencies (shedding from separated regions, for example).

The method proposed in this paper is direct unsteady U-RANS, in the temporal domain, employing spatial filtering at the interface planes to remove higher harmonics that are not of interest. Based on the problem under consideration, the user sets the filter threshold such that only frequency content below this limit passes through the interface plane. The unwanted frequencies are mixed-out, hence the new approach forms a continuum between conventional mixing planes (where no harmonics are retained across the interface) and sliding planes (where all available harmonics are preserved). The paper is organized as follows. The filtering mixing plane concept is first developed with a simple two-dimensional, single equation advection solver. Next, the entropy production during the mixing out of unwanted harmonics is demonstrated analytically. 
A strategy for the implementation of filtering mixing planes into an existing solver is then presented. Finally, the approach is demonstrated on a series of test cases of increasing complexity.

\section{REQUIREMENTS FOR FILTERING MIXING PLANES}

At the outset, we list our requirements for the filtering mixing plane approach:

1. The approach must reduce the computational work for low reduced frequency problems, as compared to the "direct unsteady" approach, by a meaningful amount. Different practitioners will have a range of views on what is "meaningful" but we define this to be an order of magnitude improvement.

2. It should be straightforward to implement the treatment within the framework of an existing time domain U-RANS code so that the new method can form a continuous arc of modelling from conventional mixing-plane to the traditional sliding interpolation plane.

3. The principal user input should be the number of harmonics, $n_{\text {harm }}$, retained through the filtering mixing plane. No further modelling parameters or source terms should be required.

\section{DEVELOPMENT OF THE CONCEPT}

\section{Introduction}

Before discussing the implementation of the filtering mixing plane technique in a turbomachinery U-RANS code, some initial concept development can be done using a simple, one equation, two-dimensional advection solver. In this section we will test the fundamental premise governing the filtering mixing plane strategy, that the size of the time step required scales with the time period of the unsteadiness. Transport of a sinusoidal "distortion" and a Gaussian "wake" is presented, followed by a combination of both of these perturbations.

\section{D Advection Solver and Test Case}

The advection equation for $f$ in integral conservation form is,

$$
\frac{d}{d t} \int_{\mathrm{V}} f d V+\int_{\mathrm{S}} f \mathbf{v} \cdot \mathbf{d} \mathbf{A}=0
$$

where the first integral is over the control volume, the second is over the surface bounding the control volume, $\mathbf{v}$ is the velocity vector and $\mathbf{d A}$ is the area vector. In the cases presented here, the velocity field is fixed. In anticipation of the target compressible flow solver, TBLOCK [5], we use a second order vertex storage scheme with quadrilateral cells. Jameson's dual time stepping approach [6] is used to discretise the time derivative (with a second-order, 3-point backwards difference stencil) and Denton's explicit "scree" scheme [7] is used to drive the inner iterations to convergence.

The test case used to develop the concept comprises three blocks, shown as a schematic in Fig. 1. Blocks 1 and 3 are stationary, but block 2 moves at speed $U$, simulating a rotor row between two stator rows. The upper and lower edges of the domain are periodic. There are $101 \times 51$ uniformly spaced grid points in each block. The scalar $f$ is initially set to 
zero everywhere and a profile of $f$ is specified at the inlet boundary. The velocity field $\mathbf{v}$ (in the absolute frame) is fixed to $<1,0>$ throughout, so we anticipate that the profile of $f$ will advect straight though the domain, without deflection.

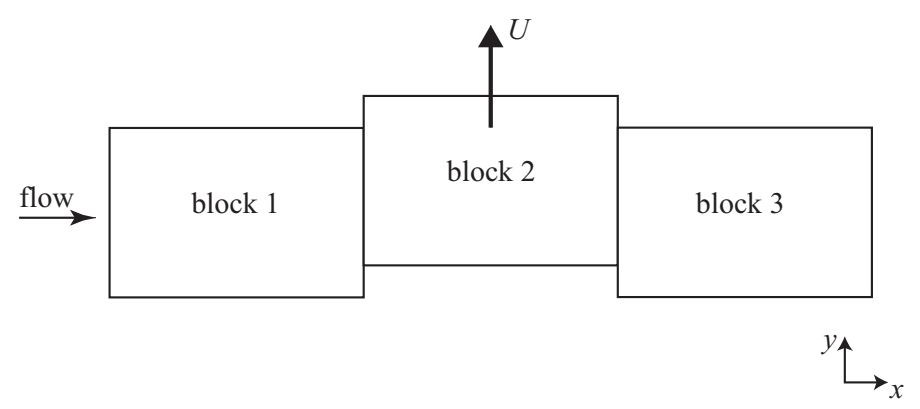

Fig. 1: Schematic of 3-block single advection equation test case domain

\section{Sinusoidal Profile}

To assess the number of time steps per period, $N_{\Delta t}$, required to resolve a sinusoidal scalar profile, four computations were run with different values of $N_{\Delta t}$. Figure 2 suggests that at least 25, and probably 50, time steps per period are required. At values of $N_{\Delta t}$ less than 25, the sinusoidal disturbance mixes and the phase (vertical alignment in Fig. 2) is shifted. The problems seen at these low values of $N_{\Delta t}$ occur in block 2, where the flow is unsteady; they are caused by the dispersion error of the second order, 3-point, approximation to the time derivative.

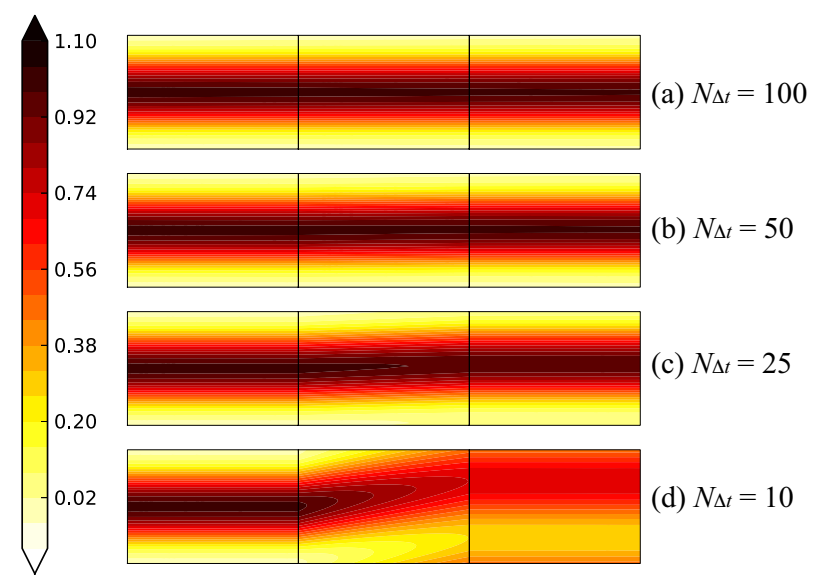

Fig. 2: Transport of a sinusoidal inlet profile through the 3-block advection equation domain

\section{Gaussian Profile}

A wake is modelled using a Gaussian profile of $f$ at the inlet of the computations shown in Fig. 3. The Gaussian profile is composed of a range of spatial frequencies. Based on the sinusoidal profile results, we expect $N_{\Delta t}=25$ will be sufficient to resolve the $1^{\text {st }}$ harmonic (wavelength equal to the distance between the periodic boundaries of the computation) but higher harmonics will be mixed and subject to a shift in phase. The exit profiles in Fig. 4 show that this is the case and that, for this simulated wake, $N_{\Delta t}=100$ is required to transfer the wake correctly through the domain. 


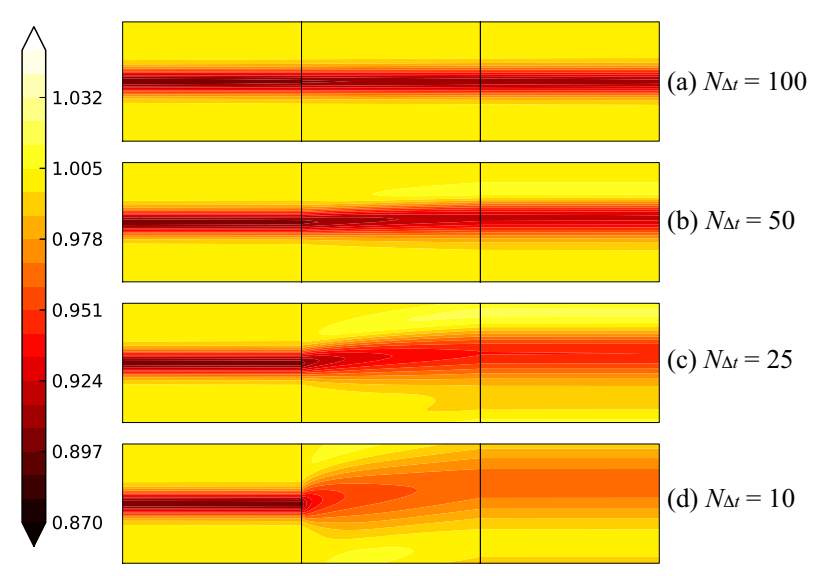

Fig. 3: Transport of a Gaussian "wake" inlet profile through the 3-block advection equation domain

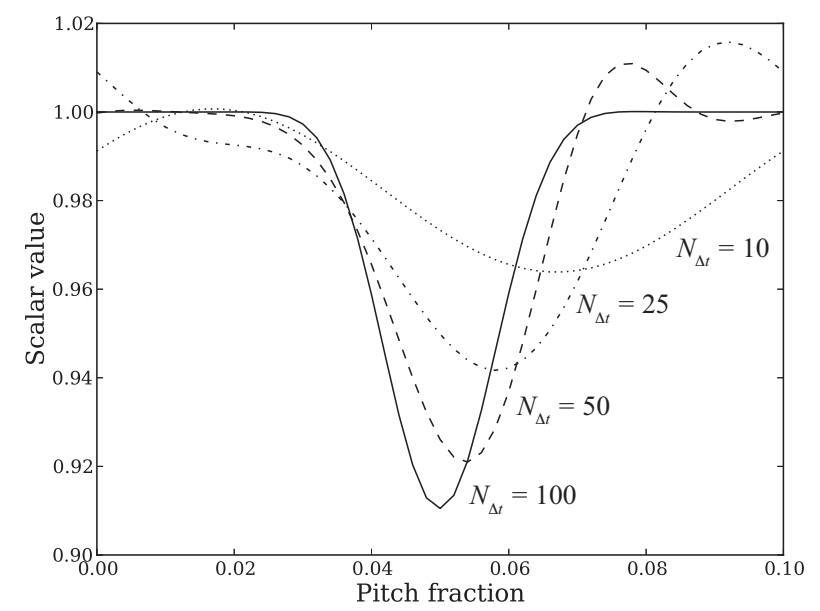

Fig. 4: Profile of scalar $f$ at the domain outlet (Gaussian "wake" at domain inlet)

\section{Combined "Distortion" and "Wakes"}

The test case is now extended by duplicating the domain 50 times in the pitchwise direction. The inlet boundary condition is now 50 sinusoidal "wake" profiles combined with a sinusoidal "distortion" of wavelength equal to the full 50 wake pitches.

The above studies have shown that 100 time steps are needed to resolve each wake, so $N_{\Delta t}=5000$ (for the full pitchwise extent of block 2 to move past a point in the absolute frame) would be required to resolve the transport of the wakes, and the distortion, through the domain. If the user is primarily concerned with distortion transfer, however, then $N_{\Delta t}=50$ would suffice. Figure 5(a) shows a simulation with $N_{\Delta t}=50$. The sinusoidal distortion is transferred through the domain, but a serious problem occurs with the wakes. Since $N_{\Delta t}=50$, at each time step, a grid point at the inlet of block 2 is always at the same position relative to the wakes leaving block 1; if a grid point at the inlet of block 2 is initially in a wake, it stays in a wake at all times. This means the wakes are stationary in the relative frame and are therefore incorrectly transferred across block 2. This is an aliasing effect. If values of $N_{\Delta t}$ close to 50 are chosen, such as those shown in Fig. 5 (b) $N_{\Delta t}=55$ and (c) $N_{\Delta t}=60$, then the wakes are no longer stationary in the relative frame, but travel circumferentially at a finite, but incorrect, speed. 


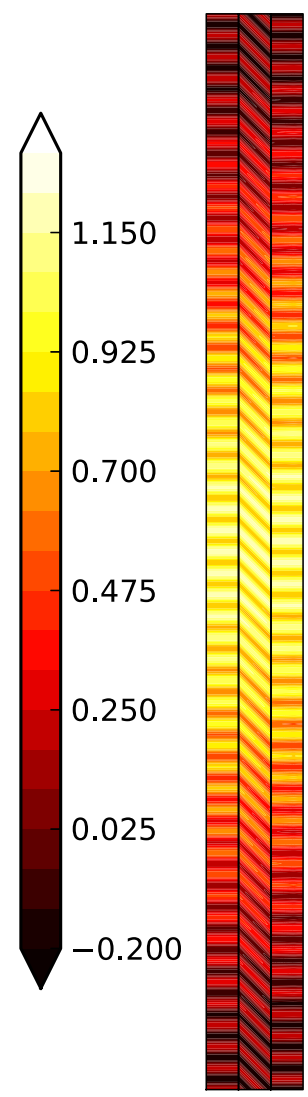

(a)

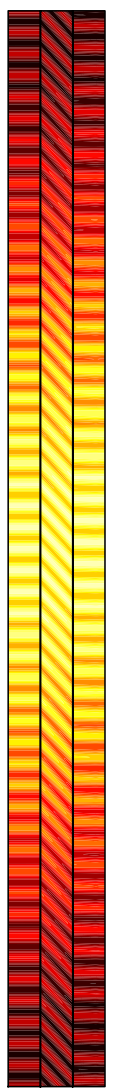

(b)

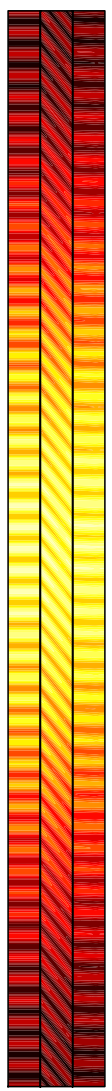

(c)

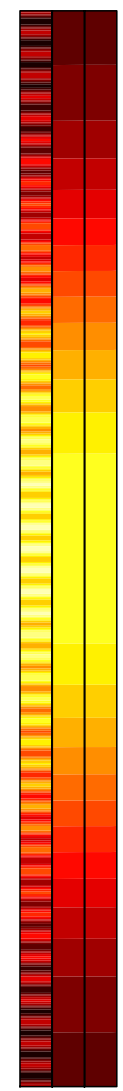

(d)

Fig. 5: Scalar transport of a long-wavelength "distortion" and 50 short wavelength "wakes": (a) $N_{\Delta t}=50$, no filter; (b) $N_{\Delta t}=55$, no filter; (c) $N_{\Delta t}=60$, no filter; (d) $N_{\Delta t}=50, n_{\text {harm }}=1$.

The aliasing problem is cured by applying a spatial filter at the block boundaries so that the wakes are removed. In Fig. 5(d), all harmonics higher than the $1^{\text {st }}$ are filtered out. The computation now proceeds without problem for $N_{\Delta t}=50$. In this demonstration, the number of inner iterations in the dual time stepping approach was fixed so that the computational effort scales with the number of time steps per period, $N_{\Delta t}$. The filtering plane allows this distortion transfer simulation to be completed with a two order of magnitude reduction in computational effort $\left(N_{\Delta t}=50\right.$ with the filtering plane compared to $N_{\Delta t}=5000$ needed in a U-RANS computation where the wakes are resolved).

\section{FLUX FILTERING AND MIXING}

The preceding section developed the case for filtering to reduce the number of time steps required in a U-RANS computation of a low reduced frequency problem while avoiding aliasing. In a turbomachinery flow solver the most appropriate choice of variables for filtering is the conservative fluxes of mass, momentum and energy. For a two-dimensional domain $(x, y)$, at an interface boundary normal to the $x$ direction, these fluxes are,

$$
\mathbf{F}=<\rho V_{x}, \rho V_{x} V_{y}, \rho V_{x}^{2}+p, \rho V_{x} h_{0}>
$$

The filtering process filters out all spatial harmonics (in the pitchwise direction) above a user-specified threshold. This is achieved by pre-multiplying $\mathbf{F}$ by the filtering matrix $\mathbf{Y}$ (a $N \times N$ matrix where $N$ is the number of pitchwise mesh points at 
the interface),

$$
\widetilde{\mathbf{F}}=\mathbf{Y} \cdot \mathbf{F}
$$

The derivation of $\mathbf{Y}$ is given in Appendix A.

Since the $0^{\text {th }}$ harmonic (the "DC level") is always retained, the total fluxes are conserved. The chosen number of retained harmonics dictates how much mixing occurs. As an example, a Gaussian $V_{x}$ wake profile ( $\rho, p$ and $T_{0}$ are all constant, $\left.V_{y}=0\right)$ is filtered analytically. The filtered profiles are shown in Fig. 6. A mixing loss coefficient can be evaluated by computing the non-dimensional drop in mass-averaged stagnation pressure, $\overline{p_{0}}$ between the non-filtered ("upstream") value, $\overline{p_{01}}$ and the filtered (“downstream”) value, $\overline{p_{02}}$ :

$$
Y_{p, \text { mix }}=\frac{\overline{p_{01}}-\overline{p_{02}}}{\overline{p_{01}}-p_{1}}
$$

The variation of $Y_{p, \text { mix }}$ with the number of harmonics retained is shown in Fig. 7. The value for a conventional mixing plane $\left(n_{\text {harm }}=0\right)$ is $Y_{p, \text { mix }}=0.014$. As the number of retained harmonics is increased, $Y_{p, \text { mix }}$ decreases monotonically until the value is negligible (less than $1 \%$ of the $n_{\text {harm }}=0$ case) for $n_{\text {harm }}>8$. Also shown in Fig. 7 is the fully mixed-out loss, i.e. the value of $Y_{p \text {,mix }}$ obtained after the filtered profile is further mixed to pitchwise uniformity. Since the filtering process conserves the fluxes of mass, momentum and energy, the mixed-out loss is unchanged as $n_{\text {harm }}$ is varied.

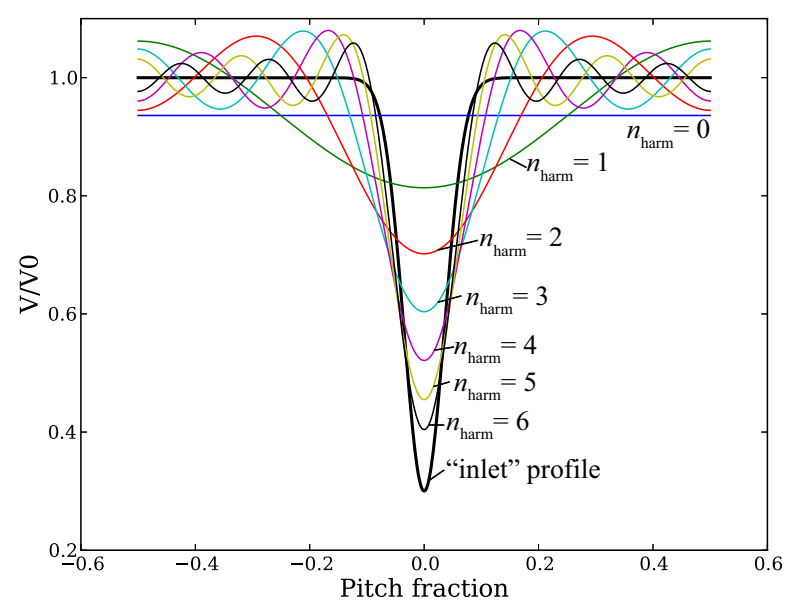

Fig. 6: Post-filtering velocity profiles of a Gaussian wake

\section{IMPLEMENTATION}

The filtering mixing plane concept has been implemented in the Denton multi-block structured U-RANS solver, TBLOCK [5]. The principal requirement is that the difference in the filtered fluxes on each side of the interface plane drives the changes to the boundary conditions for the adjacent blocks. The boundary conditions are then updated, at each iteration, until the filtered fluxes are converged.

The 3-step process followed is shown in the flow chart of Fig. 8. The steps are, for each spanwise location: 


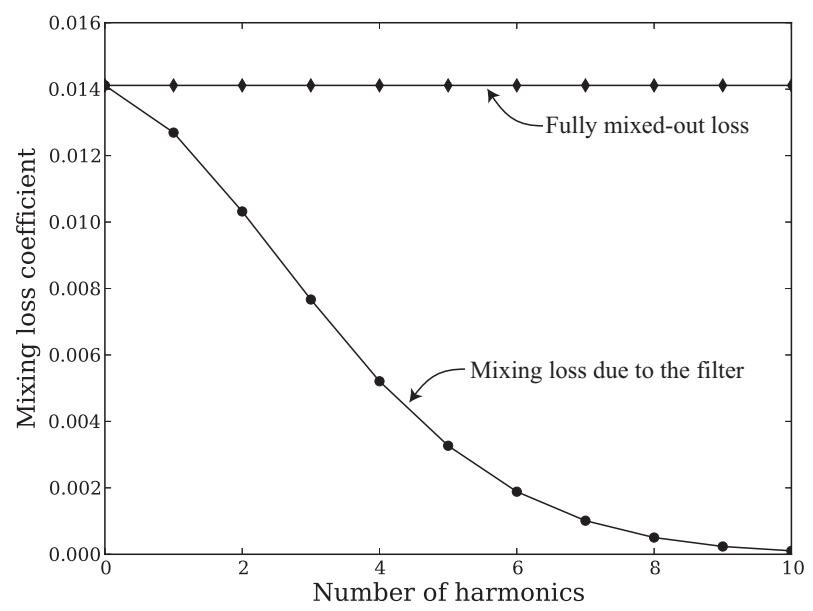

Fig. 7: Reduction of mixing loss coefficient $Y_{p \text {,mix }}$, as $n_{\text {harm }}$ is increased, of an analytic mixing calculation of a Gaussian wake. The fully mixed-out loss remains constant.

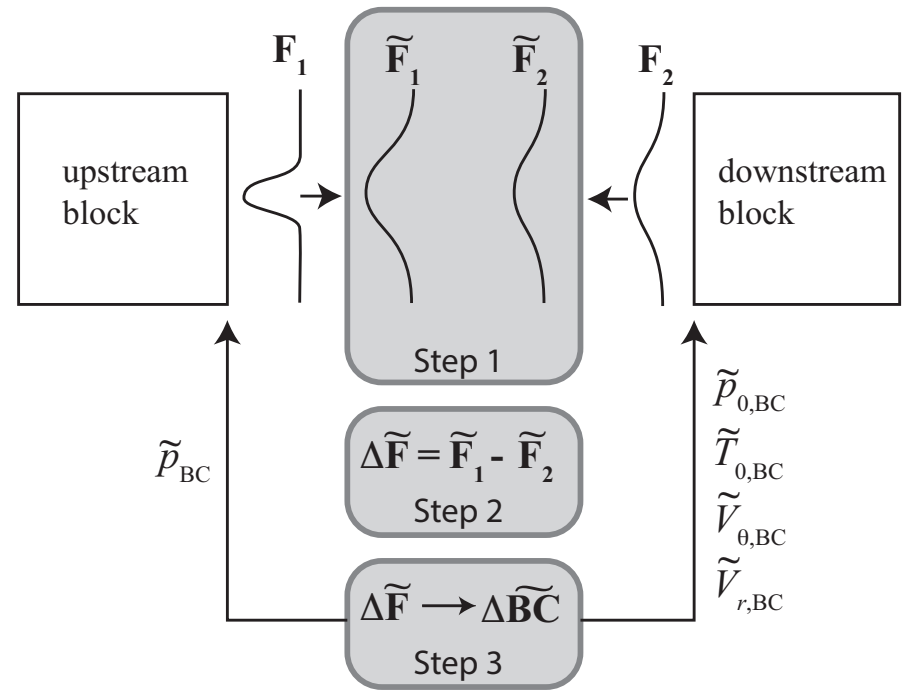

Fig. 8: Flow chart of implementation of filtering mixing planes

1. A vector of fluxes $\mathbf{F}$ is calculated for each grid node in the pitchwise direction. For a three-dimensional $(x, r, \theta)$ computation, assuming, for convenience only, that the mixing plane is normal to the axial direction $(x)$ the fluxes are:

$$
\mathbf{F}=<\rho V_{x}, \rho V_{x} V_{r}, \rho V_{x} V_{\theta}, \rho V_{x}^{2}+p, \rho V_{x} h_{0}>
$$

$\mathbf{F}$ is evaluated on the upstream $\left(\mathbf{F}_{1}\right)$ and downstream $\left(\mathbf{F}_{2}\right)$ sides of the interface. The flux vectors are filtered, using a matrix $\mathbf{Y}$ that is computed once on start-up, to obtain $\widetilde{\mathbf{F}_{\mathbf{1}}}$ and $\widetilde{\mathbf{F}_{\mathbf{2}}}$.

2. At each pitchwise grid point, the difference in the filtered flux vectors is evaluated,

$$
\Delta \widetilde{\mathbf{F}}=\widetilde{\mathbf{F}_{1}}-\widetilde{\mathbf{F}_{2}}
$$

If the blocks on either side of the interface are in relative motion, a conservative interpolation procedure is required to find $\Delta \widetilde{\mathbf{F}}$. 
3. The difference in the filtered flux vectors is transformed into a change, $\Delta \mathbf{B C}$, that is applied to the exit boundary condition of the upstream block and the inlet boundary condition of the downstream block. Before the modified boundary condition is applied, it is filtered using the same matrix $\mathbf{Y}$.

$$
\widetilde{\mathbf{B C}}=\mathbf{Y} \cdot \mathbf{B C}
$$

In this way, the downstream block sees only the filtered harmonics of the information arriving from the upstream block, and vice versa.

The process of connecting a desired change in filtered flux $\Delta \widetilde{\mathbf{F}}$ with the associated change in boundary condition $\Delta \mathbf{B C}$ (Step 3) follows the Holmes non-reflecting mixing plane treatment that is carefully described in [8]. The procedure is reproduced here for completeness, using the same notation as Holmes. The vector $\Delta \widetilde{\mathbf{F}}$ is transformed into the vector of changes to the primitive variables,

$$
\Delta \mathbf{P}=<\Delta \rho, \Delta V_{r}, \Delta V_{\theta}, \Delta V_{x}, \Delta p>
$$

by premultiplying $\Delta \widetilde{\mathbf{F}}$ by the inverse of matrix $\mathbf{A}$,

$$
\Delta \mathbf{P}=\mathbf{A}^{-1} \cdot \Delta \widetilde{\mathbf{F}}
$$

where $\mathbf{A}$ is given in Appendix B. The change in primitive variables is transformed into a change of characteristic variables $\Delta \lambda$ by the multiplying by $\mathbf{B}$ (see Appendix B):

$$
\Delta \lambda=\mathbf{B} \cdot \Delta \mathbf{P}
$$

where

$$
\Delta \lambda=<\Delta p-c^{2} \Delta \rho, \rho c \Delta V_{r}, \rho c \Delta V_{\theta}, \Delta p+\rho c \Delta V_{x}, \Delta p-\rho c \Delta V_{x}>
$$

This allows the upstream propagating characteristics to be separated from the downstream propagating characteristics. Assuming a case of positive and subsonic $V_{x}$, the change made to the upstream block's boundary condition comes only from $\Delta \lambda_{4}\left(\Delta \lambda_{1}, \Delta \lambda_{2}, \Delta \lambda_{3}, \Delta \lambda_{5}\right.$ are set to zero); the change to the boundary condition of the downstream block is made after $\Delta \lambda_{4}$ is set to zero. The change in the boundary condition variables is obtained by transforming back into the primitive set and then finally to the boundary condition variables themselves:

$$
\begin{gathered}
\Delta \mathbf{P}=\Delta \mathbf{B}^{-1} \cdot \Delta \lambda \\
\Delta \mathbf{B C}=\mathbf{U} \cdot \Delta \mathbf{P}
\end{gathered}
$$

where $\mathbf{U}$ is given in Appendix B and

$$
\Delta \mathbf{B C}=<\Delta p_{0}, \Delta T_{0}, \Delta V_{\theta}, \Delta V_{r}, \Delta p>
$$




\section{APPLICATIONS}

\section{Introduction}

Three test applications of the filtering mixing plane implementation are presented: a two-dimensional wake profile; a 1.5 stage two-dimensional compressor with inlet distortion; and a three-dimensional turbine stage with a 1:5 stator:rotor bade count ratio.

\section{Two-Dimensional Wake}

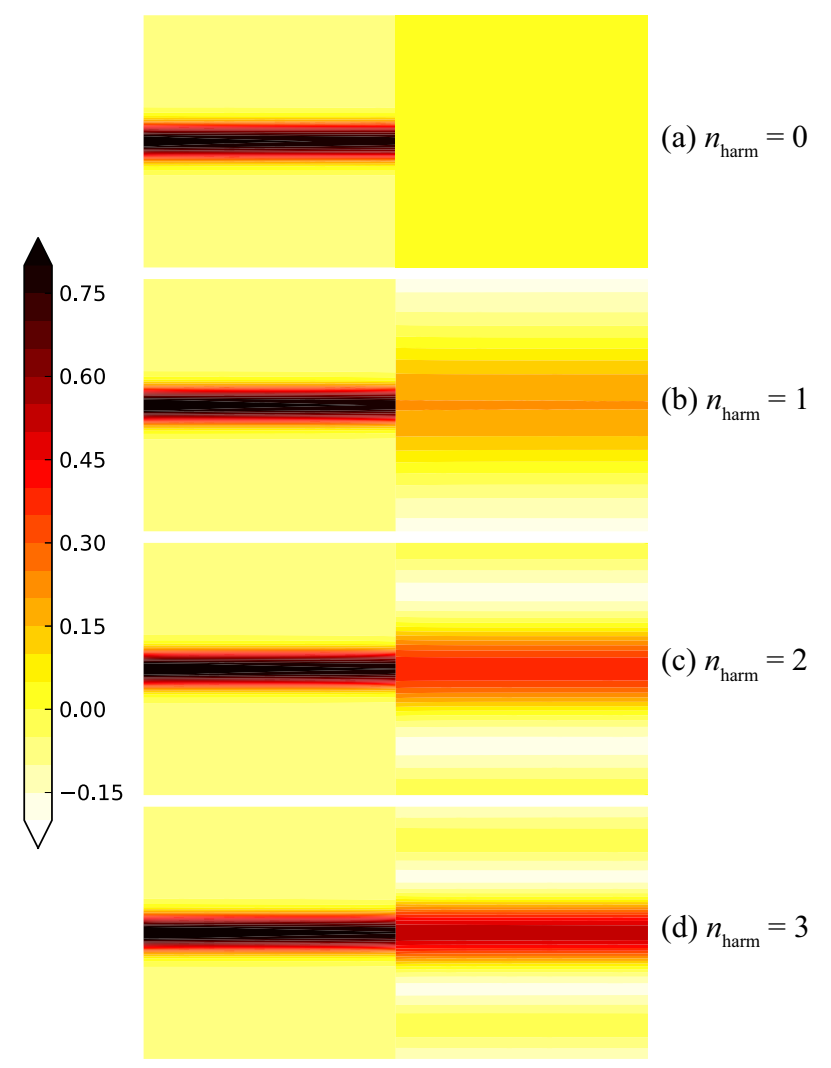

Fig. 9: Stagnation pressure loss coefficient, $Y_{p}$, for a filtering mixing plane with a 2D wake

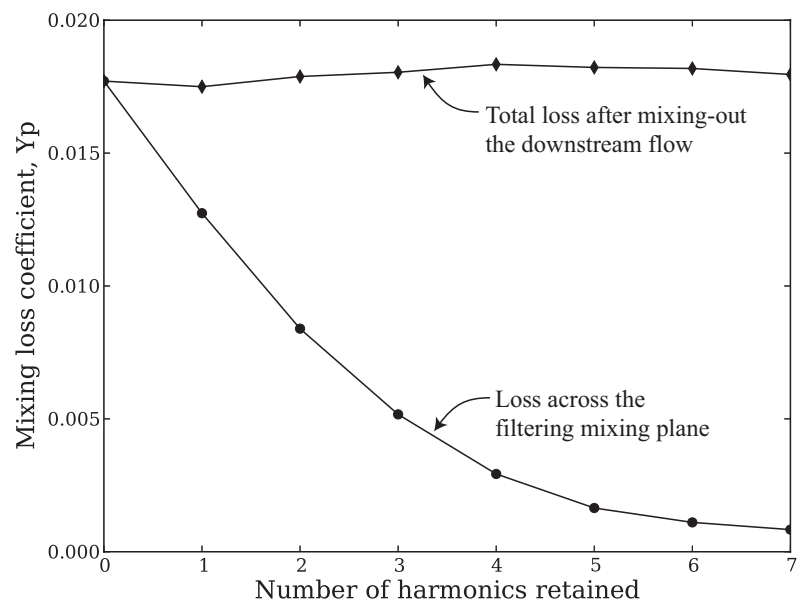

Fig. 10: Reduction of mixing loss coefficient, $Y_{p \text {, mix }}$, as number of retained harmonics is increased for 2D wake case 
This is a simple case, comprized of two blocks, designed to verify the filtering mixing plane implementation, particularly its conservation properties. The filtering mixing plane is located at the interface between the blocks. Each block has a grid comprized of $51 \times 51 \times 5$ points (axial $\times$ pitchwise $\times$ spanwise). The free stream Mach number is 0.3 and the Mach number deficit in the centre of the wake (imposed by a Gaussian profile of inlet stagnation pressure) is 0.2 .

Four cases are shown in Fig. 9, each with a different number of retained harmonics, $n_{\text {harm }}$. As the number of retained harmonics is increased, the amount of mixing that takes place at the filtering mixing plane reduces and the wake in the downstream block becomes qualitatively similar to that in the upstream block. The mass-averaged mixing loss coefficient, Fig. 10, illustrates that the quantitative behavior seen in the analytic case (Fig. 7) is reproduced with a smooth, monotonic reduction in $Y_{p, m i x}$ as $n_{\text {harm }}$ is increased. To accomplish this, the fluxes must be closely conserved at the filtering mixing plane; the mass conservation error, for example, was less than $0.0005 \%$ in all cases. The fully mixed-out line in Fig. 10 shows a small fluctuation (standard deviation is $1.5 \%$ of the mean).

\section{Two-Dimensional Compressor Stage}

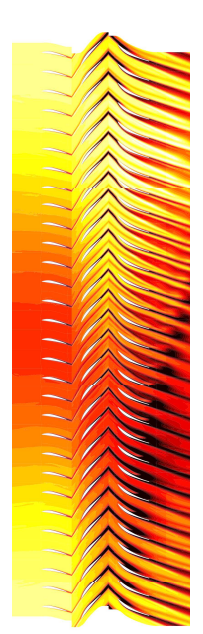

(a)

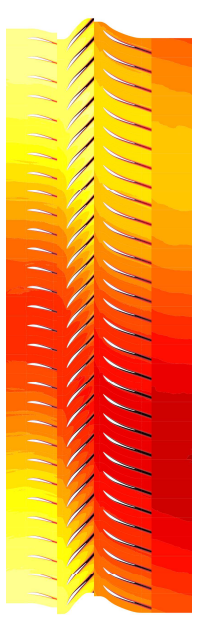

(b)

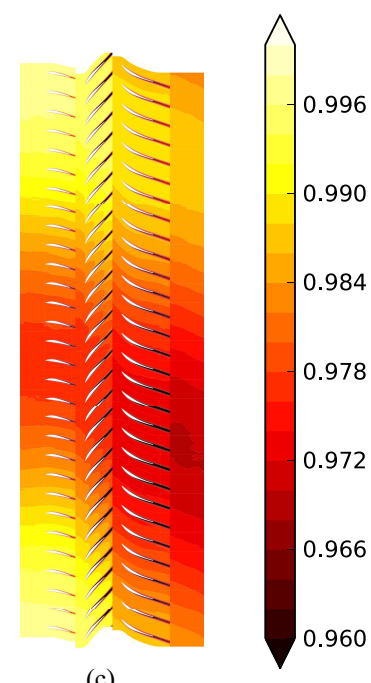

(c)

Fig. 11: 1.5 stage axial compressor with inlet distortion (full domain not shown), contours of entropy function $e^{-\Delta s / R}:(a)$ $N_{\Delta t}=30$, no filter; (b) $N_{\Delta t}=30, n_{\text {harm }}=1 ;$ (c) $N_{\Delta t}=100, n_{\text {harm }}=1$

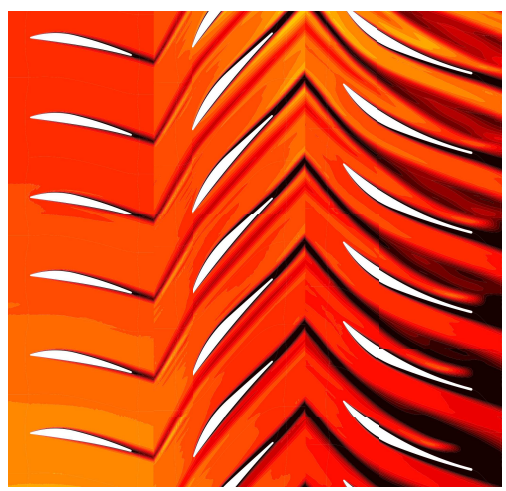

Fig. 12: Detail of $N_{\Delta t}=30$, no filter, computation of Fig. 11(a) - contours of entropy function $e^{-\Delta s / R}$. Aliasing means the computation is effectively "frozen rotor" 
Compressor inlet distortion is a classic low reduced frequency problem that the filtering mixing plane method is designed to tackle. The geometry for the present case comes from the mid-span of a low-speed research compressor designed to be representative of current industrial gas turbines [9]. A half-annulus domain is used: 30 inlet guide vanes, 29 rotor blades and 30 stators. The blade-to-blade mesh had a total of 1.3 million grid points and there were 5 points in the spanwise direction. The purpose of the computation is to capture distortion transfer and the quasi-steady behavior of the compressor. In tackling the problem using a time-step based on the unsteadiness arising from the long length-scale perturbations, the user acknowledges that the wake interaction process will not be resolved (including any "wake recovery" effects, for example) and is content to mix out the wakes at the row interfaces.

Figure 11 shows contours of entropy function, $e^{-\Delta s / R}$, for three cases: (a) $N_{\Delta t}=30$, no filter; (b) $N_{\Delta t}=30, n_{\text {harm }}=$ 1; (c) $N_{\Delta t}=100, n_{\text {harm }}=1$. The figure does not show the full domain, which extends upstream and downstream of the compressor by a distance equal to approximately one quarter of the machine circumference. The $N_{\Delta t}=30$ case exhibits a strong aliasing effect (more detail is visible in Fig. 12) at both the IGV-rotor and rotor-stator interfaces. The rotor moves by $1 / 30^{\text {th }}$ of the total pitchwise extent of the domain at each time step. Since there are 30 IGVs, the IGV wakes appear stationary in the rotor-relative frame. As there are 29 rotor blades, the rotor wakes move slowly across a stator pitch, taking half of a revolution to traverse a single stator pitch, in an entirely non-physical manner. The $n_{\text {harm }}=1$ spatial filter in Fig. 11(b) eliminates the wakes but tests with the simple advection solver indicated that $N_{\Delta t}=50$ is required to resolve even a sinusoidal perturbation. To illustrate this, $N_{\Delta t}=100$ is used in Fig. 11(c) and there is a discernible phase shift in the transferred distortion as compared to Fig. 11(b).

In performing these computations, the same convergence criterion was applied to the inner dual time stepping iterations for the filtered cases as would be used in a conventional sliding plane computation. It was observed that the $N_{\Delta t}=100$ case typically required twice the number of inner iterations compared to a wake-resolving $N_{\Delta t}=3000$ case. The total number of steps (physical time steps multiplied by inner iterations) was, therefore, an order of magnitude smaller for the $N_{\Delta t}=100$ case. This test application has demonstrated the computational efficiency of the filtering mixing plane technique when applied to a low reduced frequency problem ( $\beta \approx 0.05$ ). We now turn to a configuration in turbine aerodynamics for which the present method is also appropriate.

\section{Three-Dimensional Turbine Stage}

Low aspect ratio vanes, or mid-frame struts, are a feature of many aero-engine turbines. It is not uncommon for the vane pitch to be 5 times that of the downstream blade rows, as shown in Fig. 13. In such a case, the reduced frequency of the rotor will be, approximately $\beta \approx 0.4$.

The vane loss core is of the order of a rotor pitch in circumferential extent and it is known that this leads to secondary flows at rotor exit that are both stationary in the absolute frame and also, due to the low reduced frequency, quasi-steady. The interaction of such secondary flows with the downstream stages is of interest but the computations are costly as the rotor wakes require of order 100 time steps per rotor passing period, $N_{\Delta t}=500$, to resolve the rotor wakes in the downstream stage.

A sample turbine stage was created using the highly loaded turbine profile tested by Harrison [10]. The total mesh count 


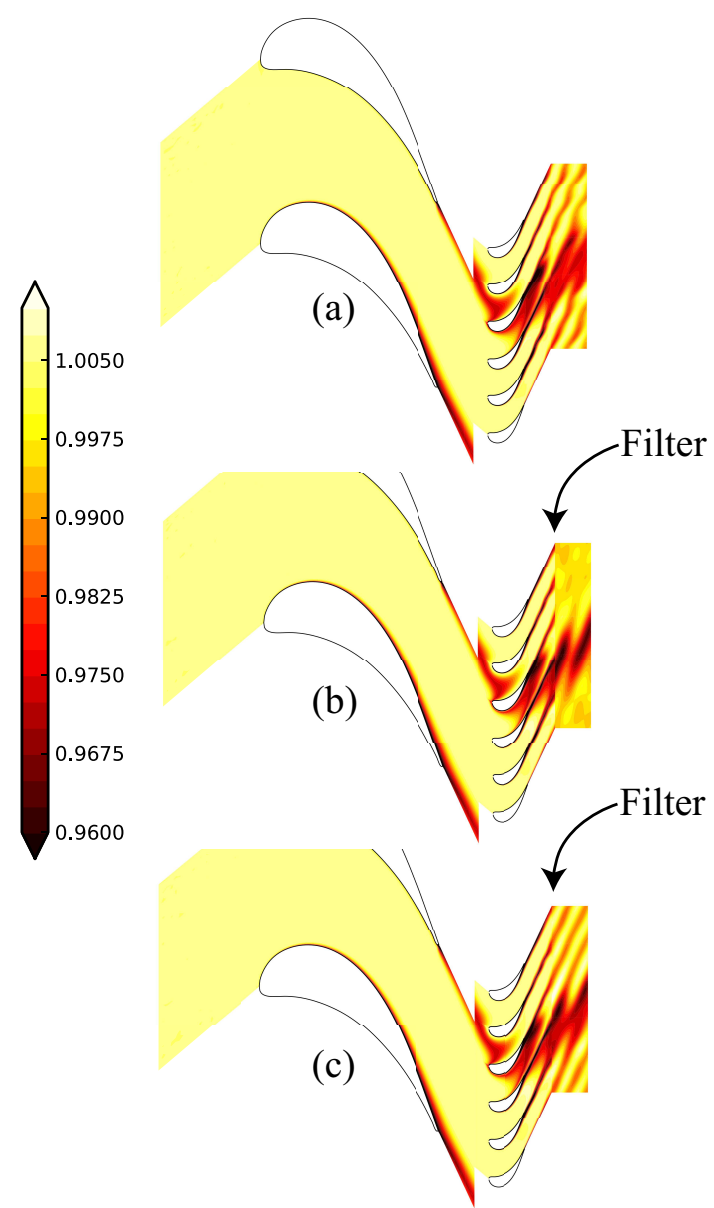

Fig. 13: 3D turbine stage with low aspect ratio vane, mid-span entropy $e^{-\Delta s / R}$ : (a) $N_{\Delta t}=100$, no filter; (b) $N_{\Delta t}=100$, $n_{\text {harm }}=4 ;(\mathrm{c}) N_{\Delta t}=100, n_{\text {harm }}=5$
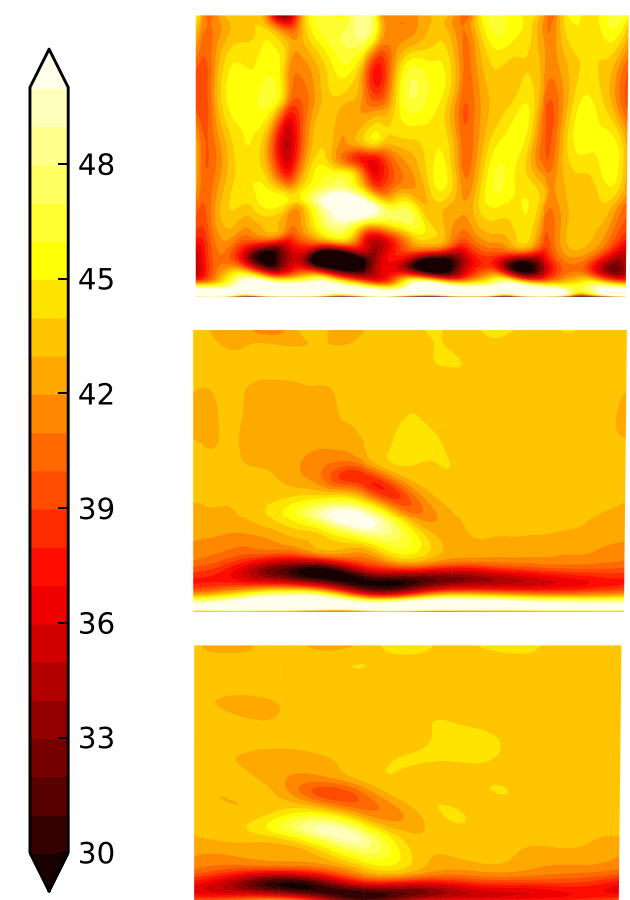

(a)

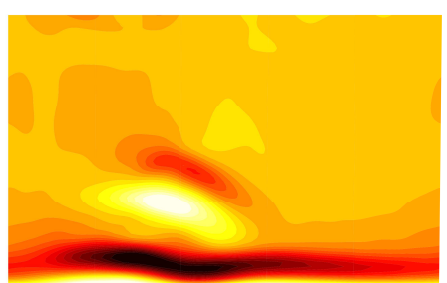

(b)

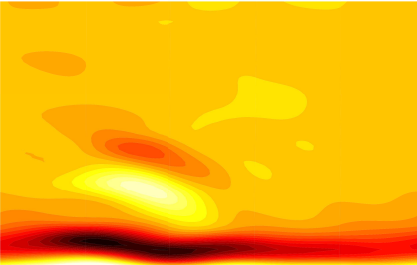

(c)

Fig. 14: 3D turbine stage rotor exit absolute swirl angle (degrees): (a) $N_{\Delta t}=100$, no filter; (b) $N_{\Delta t}=100$, no filter, timeaveraged; (c) $N_{\Delta t}=100, n_{\text {harm }}=4$, time-averaged 
was 7 million nodes and covers half of the span (the upper wall is inviscid). For this case, a conventional sliding plane was employed between the vane and rotor, and a filtering mixing plane between the rotor and the (stationary) exit block. The objective is to demonstrate that the filter can remove the rotor wakes, leaving an accurate representation of the vane wake and the associated secondary flows. This would allow the downstream stages to be computed with a time step based on the vane pitch $\left(N_{\Delta t}=100\right)$ rather than on the rotor pitch $\left(N_{\Delta t}=500\right)$.

Fig. 13 shows the transport of the vane wake through the rotor row using entropy as a marker. All three computations use $N_{\Delta t}=100$. Fig. 13(a) employs a sliding plane at both interfaces. Fig. 13(b) uses a filtering mixing plane at the second interface and retains the leading four harmonics, $n_{\text {harm }}=4$. Since there are 5 rotors per vane passage, this means that the rotor wakes are mixed-out (hence the jump in entropy) and are not present in the exit block. With one more harmonic, Fig. 13(c), the rotor wakes are present, albeit the higher harmonics are mixed out. The loss coefficient across the filtering mixing plane for the $n_{\text {harm }}=4$ case is $70 \%$ of the value for a $n_{\text {harm }}=0$ (conventional mixing plane) computation; the remaining $30 \%$ arises from the mixing of the rotor exit flow features associated with the interaction of the rotor with the vane.

The exit flow field is depicted, using contours of absolute swirl angle, in Fig. 14. An instantaneous snapshot of the sliding plane computation, Fig. 14(a), shows the 5 rotor wakes, and 5 rotor hub secondary flow vortices (zones of overturning at the endwall with under-turning at approximately $15 \%$ of span). The time-average of this computation is plotted in Fig. 14(b), and it is clear that the time-average of the $n_{\text {harm }}=4$ calculation, Fig. 14(c), is a close match.

If the interaction of the low aspect ratio vane with the downstream stage (not computed here) is of primary interest, the $n_{\text {harm }}=4$ filtering mixing plane could be used with $N_{\Delta t}=100$ rather than the $N_{\Delta t}=500$ that would be required without a filter.

\section{CONCLUSIONS}

1. A filtering mixing plane treatment has been developed with the aim of an order of magnitude reduction in computational work for low reduced frequency analysis of flows in turbomachines.

2. The filtering mixing plane treatment mixes out the unwanted higher harmonics at the interface plane. The number of retained harmonics can be set by the user so that a continuum is formed between zero retained harmonics (conventional mixing plane) and all harmonics retained (conventional sliding plane). Having eliminated the higher spatial harmonics which the user deems are of secondary importance, a larger time step can then be chosen to resolve the low reduced frequency of interest.

3. The proposed implementation of the filtering mixing plane is based on the core principal that the difference in the filtered conservative fluxes on each side of the interface drives the changes to the boundary conditions in the adjacent blocks.

4. The filtering mixing plane was demonstrated on three test cases: a two-dimensional wake, a two-dimensional 1.5 stage compressor with inlet distortion and a three-dimensional turbine stage with a low aspect ratio vane. The method showed a smooth, monotonic reduction in wake mixing loss as the number of retained harmonics is increased. Computational work reductions of up to a factor of 5 were seen for the turbine case and more than an order of magnitude for the compressor case. 


\section{Acknowledgements}

The authors are grateful to Professors L. He and J.D. Denton for their comments during this work. Thanks are also given to MHI for permission to use the compressor geometry.

\section{NOMENCLATURE}

\begin{tabular}{cl}
$c$ & Chord \\
$f$ & Frequency or passive scalar \\
$h$ & Enthalpy \\
$n_{\text {harm }}$ & Number of retained harmonics \\
$N_{\Delta t}$ & Number of time steps per period \\
$p$ & Pressure \\
$T$ & Temperature \\
$U$ & Blade speed \\
$V$ & Velocity \\
$\beta$ & Reduced frequency \\
$\lambda$ & Wavelength or characteristic variable \\
$\phi$ & Flow coefficient \\
$\rho$ & Density \\
$<\ldots>$ & Vector \\
$\mathbf{v}$ & Velocity vector \\
$\mathbf{d A}$ & Area vector \\
$\mathbf{F}$ & Vector of fluxes \\
$\mathbf{P}$ & Vector of primitive variables \\
$\mathbf{B C}$ & Vector of boundary conditions \\
\hline
\end{tabular}

subsripts:

$x, r, \theta \quad$ Axial, radial, tangential

$0 \quad$ Stagnation

\section{References}

[1] Michellasi, V., Chen, L., Pichler, R., Sandberg, R., and Bhaskaran, R., 2015. "High-fidelity simulations of low-pressure turbines: Effect of flow coefficient and reduced frequency on losses”. ASME J. Turbomach., 138(11).

[2] He, L., and Ning, W., 1998. "An efficicient approach for analysis of unsteady viscous flows in turbomachines". AIAA J., $36(11)$.

[3] Hall, K., Thomas, J., and Clark, W., 2002. "Computation of unsteady nonlinear flows in a cascade using a harmonic balance technique". AIAA J., $\mathbf{4 0}(5)$.

[4] He, L., 2010. "Fourier methods for turbomachinery applications". Progress in Aerospace Sciences, 46. 
[5] Rosic, B., Denton, J., and Pullan, G., 2006. "The importance of shroud leakage modelling in multistage turbine flow calculations". ASME J. Turbomach., 128(4).

[6] Jameson, A., 1991. "Time dependent calculations using multigrid, with applications to unsteady flows past airfoils and wings". AIAA 91-1596.

[7] Denton, J., 2002. "The effects of lean and sweep on transonic fan performance: A computational study". TASK Quarterly, 6(1), pp. 1-17.

[8] Holmes, D., 2008. "Mixing planes revisited: A steady mixing plane approach designed to combine high levels of conservation and robustness". ASME IGTI Turbo Expo, GT2008-51296.

[9] Grimshaw, S., Pullan, G., and Walker, T., 2015. "Bleed-induced distortion in axial compressors". ASME J. Turbomach., 137(10).

[10] Harrison, S., 1992. "The influence of blade lean on turbine loss". ASME J. Turbomach., 114(1).

\section{APPENDIX A}

In the following, the circumferential grid spacing is assumed to be uniform, for simplicity. The development of the spatial filter employed in this paper is based on the mathematics of the discrete Fourier series. A function that is assumed to have a known circumferential periodicity (the full circumferential extent, or "sector size", of the domain) can be expressed by the discrete Fourier series,

$$
x_{n}=\frac{1}{N} \sum_{k=0}^{N-1}\left(A_{k} \cos \frac{2 \pi k n}{N}-B_{k} \sin \frac{2 \pi k n}{N}\right)
$$

where $x_{n}$ is the function at a discrete location $n$, and $N$ is the number of points at which $x$ is defined. The coefficients $A_{k}$ and $B_{k}$, which will also be functions of radius, are the Fourier coefficients. Since $x_{n}$ is real, $A_{k}$ and $B_{k}$ must also be real. $A_{k}$ and $B_{k}$ can be obtained from the $N$ known values of $x$ using

$$
\begin{aligned}
A_{k} & =\sum_{m=0}^{N-1} x_{m} \cos \frac{2 \pi k m}{N} \\
B_{k} & =-\sum_{m=0}^{N-1} x_{m} \sin \frac{2 \pi k m}{N}
\end{aligned}
$$

The filter is developed by omitting spatial components from Equation (A1) above a pre-determined value. At a particular location, the filtered variable $\widetilde{x}_{n}$ is then given by

$$
\widetilde{x}_{n}=\frac{1}{N} \sum_{k=0}^{M-1}\left(A_{k} \cos \frac{2 \pi k n}{N}-B_{k} \sin \frac{2 \pi k n}{N}\right)
$$

where $M$ is the number of spatial components to be retained and can take values between 1 and $N$ (note that this includes the "zero", or DC, harmonic). The choice for the value of $M$ is based on the spatial length-scales to be resolved and is set by the 
user. Introducing Equations (A2) and (A3) into Equation (A4) yields

$$
\widetilde{x}_{n}=\frac{1}{N} \sum_{m=0}^{N-1} x_{m}\left[\sum_{k=0}^{M-1} \cos \frac{2 \pi k}{N}(n-m)\right]=\frac{1}{N} \sum_{m=0}^{N-1} x_{m} R(n-m)
$$

where the series $R(n-m)$ is the response function associated with the filtering process. $R(n-m)$ may be expressed as

$$
R(n-m)=\frac{\cos \left(\frac{\pi(M-1)(n-m)}{N}\right) \sin \left(\frac{\pi M(n-m)}{N}\right)}{\sin \left(\frac{\pi(n-m)}{N}\right)}
$$

The filtered variable at location $n, \widetilde{x}_{n}$ is thus related to the known values of the unfiltered variable $x$ via

$$
\widetilde{x}_{n}=\frac{1}{N} \sum_{m=0}^{N-1} x_{m} R(n-m)
$$

Equation (A7) is then used to populate the elements of the filter matrix $\mathbf{Y}$ such that

$$
\widetilde{x}=\mathbf{Y} \cdot x
$$

Note that the response function $R$ retains all harmonics, a U-RANS sliding plane treatment, when $M=N$. When $M=1$, $\widetilde{x}$ is the pitch-wise average of $x$ and the interface becomes a conventional mixing plane. The response function, and hence the full matrix $\mathbf{Y}$ can be computed prior to the start of the simulation and is solely a function of the spatial circumferential length-scales to be resolved and the number of grid points in the circumferential direction. Application of the filtering process during a simulation involves a matrix multiplication, for which efficient means of implementation have been developed.

\section{APPENDIX B}

The matrix that transforms changes in the primitive variables $\Delta \mathbf{P}$ to changes in the flux variables $\Delta \mathbf{F}$ is:

$$
\mathbf{A}=\left[\begin{array}{ccccc}
v_{x} & 0 & 0 & \rho & 0 \\
v_{x} v_{r} & \rho v_{x} & 0 & \rho v_{r} & 0 \\
v_{x} v_{\theta} & 0 & \rho v_{x} & \rho v_{\theta} & 0 \\
v_{x}^{2} & 0 & 0 & 2 \rho v_{x} & 1 \\
v_{x} h_{0}+\left.\rho v_{x} \frac{\partial h}{\partial \rho}\right|_{p} & \rho v_{x} v_{r} & \rho v_{x} v_{\theta} & \rho h_{0}+\rho v_{x}^{2} & \left.\rho v_{x} \frac{\partial h}{\partial p}\right|_{\rho}
\end{array}\right]
$$

The matrix that transforms changes in the primitive variables $\Delta \mathbf{P}$ to changes in the characteristic variables $\Delta \lambda$ is:

$$
\mathbf{B}=\left[\begin{array}{ccccc}
-c^{2} & 0 & 0 & 0 & 1 \\
0 & \rho c & 0 & 0 & 0 \\
0 & 0 & \rho c & 0 & 0 \\
0 & 0 & 0 & \rho c & 1 \\
0 & 0 & 0 & -\rho c & 1
\end{array}\right]
$$


The matrix that transforms changes in the primitive variables $\Delta \mathbf{P}$ to changes in the boundary condition variables $\Delta \mathbf{B C}$ is:

$$
\mathbf{U}=\left[\begin{array}{ccccc}
\left.\frac{\partial h}{\partial \rho}\right|_{p} & v_{r} & v_{\theta} & v_{x} & \left.\frac{\partial h}{\partial p}\right|_{\rho} \\
\left.\frac{\partial s}{\partial \rho}\right|_{p} & 0 & 0 & 0 & \left.\frac{\partial s}{\partial p}\right|_{\rho} \\
0 & 0 & 1 & 0 & 0 \\
0 & 1 & 0 & 0 & 0 \\
0 & 0 & 0 & 0 & 1
\end{array}\right]
$$

